\title{
Conserving adaptive potential: lessons from Tasmanian devils and their transmissible cancer
}

\author{
Paul A. Hohenlohe ${ }^{1}$ (D $\cdot$ Hamish I. McCallum ${ }^{2} \cdot$ Menna E. Jones ${ }^{3} \cdot$ Matthew F. Lawrance $^{4} \cdot$ Rodrigo K. Hamede $^{3}$. \\ Andrew Storfer ${ }^{4}$
}

Received: 7 September 2018 / Accepted: 9 February 2019 / Published online: 14 February 2019

(c) Springer Nature B.V. 2019

\begin{abstract}
Maintenance of adaptive genetic variation has long been a goal of management of natural populations, but only recently have genomic tools allowed identification of specific loci associated with fitness-related traits in species of conservation concern. This raises the possibility of managing for genetic variation directly relevant to specific threats, such as those due to climate change or emerging infectious disease. Tasmanian devils (Sarcophilus harrisii) face the threat of a transmissible cancer, devil facial tumor disease (DFTD), that has decimated wild populations and led to intensive management efforts. Recent discoveries from genomic and modeling studies reveal how natural devil populations are responding to DFTD, and can inform management of both captive and wild devil populations. Notably, recent studies have documented genetic variation for disease-related traits and rapid evolution in response to DFTD, as well as potential mechanisms for disease resistance such as immune response and tumor regression in wild devils. Recent models predict dynamic persistence of devils with or without DFTD under a variety of modeling scenarios, although at much lower population densities than before DFTD emerged, contrary to previous predictions of extinction. As a result, current management that focuses on captive breeding and release for maintaining genome-wide genetic diversity or demographic supplementation of populations could have negative consequences. Translocations of captive devils into wild populations evolving with DFTD can cause outbreeding depression and/or increases in the force of infection and thereby the severity of the epidemic, and we argue that these risks outweigh any benefits of demographic supplementation in wild populations. We also argue that genetic variation at loci associated with DFTD should be monitored in both captive and wild populations, and that as our understanding of DFTD-related genetic variation improves, considering genetic management approaches to target this variation is warranted in developing conservation strategies for Tasmanian devils.
\end{abstract}

Keywords Captive breeding $\cdot$ Conservation genomics $\cdot$ Emerging infectious disease $\cdot$ Supplementation $\cdot$ Wildlife cancer

Electronic supplementary material The online version of this article (https://doi.org/10.1007/s10592-019-01157-5) contains supplementary material, which is available to authorized users.

Paul A. Hohenlohe

hohenlohe@uidaho.edu

Andrew Storfer

astorfer@wsu.edu

1 Department of Biological Sciences, Institute for Bioinformatics and Evolutionary Studies, University of Idaho, Moscow, ID 83843, USA

2 Environmental Futures Research Institute, Griffith University, Brisbane, QLD 4111, Australia

3 School of Biological Sciences, University of Tasmania, Hobart, TAS 7001, Australia

4 School of Biological Sciences, Washington State University, Pullman, WA 99164, USA

\section{Introduction}

The maintenance or enhancement of genetic diversity has long been a focus of conservation efforts to manage declining, threatened or endangered species (Soulé and Wilcox 1980). Early efforts focused on ensuring a minimum effective population size to prevent loss of alleles via genetic drift and avoidance of demographic processes that could lead to inbreeding (Gilpin and Soulé 1986; Lande 1995). The development of genetic tools such as microsatellites allowed direct monitoring of genetic diversity and facilitated more active genetic management, such as genetic rescue, which involves introducing genetic variation into a threatened population to increase population fitness (Frankham 2015; Whiteley et al. 2015). For example, translocations 
likely prevented extinction and even facilitated recovery in the Florida panther, which suffered from inbreeding depression prior to introduction of animals from Texas (Johnson et al. 2010).

Whereas management of overall genetic diversity is a general strategy for maximizing evolutionary potential in the face of uncertain environmental change (Gilpin and Soulé 1986), we are now in the midst of an era of urgent and specific threats coming from anthropogenic global change. With the advent of genomic techniques, researchers can identify loci responsible for reduced fitness (Kardos et al. 2016), associated with variation in phenotypic traits (Santure and Garant 2018), or associated with adaptive differentiation among populations and adaptation to specific environmental factors (Rellstab et al. 2015; Hoban et al. 2016; Storfer et al. 2018b). Numerous studies have identified such loci in natural populations (reviewed by Luikart et al. 2018), and Bay et al. (2018) provide an approach for using genomic information on adaptive loci in predictive modeling of species responses to specific and immediate threats, such as climate change or emerging infectious diseases. However, when and how to tailor active conservation efforts based on information about particular loci versus overall genetic variation remains a challenging question.

Tasmanian devils (Sarcophilus harrisii) are a prime example of a species facing a specific threat. Once native to mainland Australia and the island of Tasmania, these marsupial carnivores were restricted to the island state of Tasmania by the time of European colonization (BrünicheOlsen et al. 2018). Devils appear to have experienced genetic bottlenecks prior to the contraction of their distribution to Tasmania, which affected genetic diversity at immunerelated (Morris et al. 2013) and neutral loci, likely resulting from climatic changes associated with extreme El Niño events 3000-5000 years ago and the glacial maximum more than 20,000 years ago (Brüniche-Olsen et al. 2014). Thus far, all genetic studies of devils have shown low genetic diversity in microsatellites (Jones et al. 2004; Lachish et al. 2011; Brüniche-Olsen et al. 2014), mitochondrial genomes (Miller et al. 2011; Brüniche-Olsen et al. 2018), MHC class I (Siddle et al. 2010) and class II (Cheng et al. 2012) loci, and RADseq-derived SNPs (Hendricks et al. 2017). Since the mid-1990s, Tasmanian devil populations have declined progressively island-wide as a result of the appearance and spread of devil facial tumor disease (DFTD), a transmissible cancer (Pearse and Swift 2006; Murchison 2009). Transmitted by biting during common social interactions among devils, DFTD is almost always fatal (McCallum et al. 2009; Hamede et al. 2013). In just over 20 years, this clonal cell line has spread approximately $95 \%$ of the way across the devil's geographic range, causing localized declines often exceeding $90 \%$ and a species-wide decline of approximately 80\% (McCallum et al. 2009; Hamede et al. 2015; Lazenby et al. 2018; Storfer et al. 2018a). The severity of this outbreak led to predictions of complete extinction of devils, based on compartmental epidemiological models with frequency-dependent transmission of DFTD (McCallum et al. 2009). However, no local extinctions have been documented (Lazenby et al. 2018; Storfer et al. 2018a).

\section{Recent findings in Tasmanian devils and DFTD}

A number of recent studies have provided a better understanding of the impacts of DFTD on devil populations and insights into potential future outcomes (Storfer et al. 2018a; Russell et al. 2018). A large amount of research has focused on the physiological and immunological responses of individual devils to DFTD infection (e.g., Siddle et al. 2013; Brown et al. 2016) and genetic changes in the tumor cell line (e.g., Pearse et al. 2012; Ujvari et al. 2014); here we focus on genetic variation and the potential population responses to disease.

Recent genomic studies have found evidence of both genetic variation for DFTD-related phenotypes and rapid evolution of devils at loci with functions potentially related to DFTD resistance and transmission (see Supplemental Table S1 for a list of the candidate genes identified by these studies). Epstein et al. (2016) scanned 90,000 SNP loci for multiple signatures of selective sweeps (large changes in allele frequencies pre- and post-disease, increases in linkage disequilibrium, and time-series analyses) in three independent devil populations across a wide geographic area. Strong support was found for rapid evolution (in 4-6 generations) in two small genomic regions containing seven candidate genes, five of which were associated with immune and cancer-related functions (Epstein et al. 2016). In a second study, Hubert et al. (2018) re-analyzed the same dataset using a maximum likelihood approach and extensive functional annotations, and they found evidence for responses to selection in 97 genomic regions containing 148 protein coding genes. Nearly all of these loci with human orthologs were linked with cancer, and many with behavior (Hubert et al. 2018). More directly, a genome-wide association study (GWAS) of 624 devils at nearly 16,000 SNP loci explained much of the phenotypic variance for survival of females with DFTD (> 80\%) and female case-control (>61\%) (Margres et al. 2018a). Notably, for female survival a large proportion of the variance $(>61 \%)$ was explained by relatively few $(\sim 5)$ large-effect SNP loci.

Remarkably, a small but growing number (fewer than 20) of devils have recently shown tumor regression or even complete tumor disappearance after DFTD infection (Pye et al. 2016a; Wright et al. 2017). A comparative genomic study of devils with tumor regression versus those that succumbed 
to DFTD showed evidence that three genes involved in the regression process likely stimulate angiogenesis in cancer metastases, perhaps enabling increased tumor vascularization to enable lymphocyte penetration (Wright et al. 2017). A second comparative genomic study suggested that regulatory changes in gene expression were involved (Margres et al. 2018b). Additionally, wild devils have recently shown evidence of an apparently effective immune response (Pye et al. 2016a) with circulating lymphocytes that infiltrate the tumor, as well as circulating antibodies against DFTD.

Despite models predicting devil extinction (McCallum et al. 2009), the longest diseased populations persist (Lazenby et al. 2018), and more recent modeling efforts are beginning to explain this observation. Based on long-term field study of the West Pencil Pine population located near Cradle Mountain, Wells et al. (2017) found that force of infection, the rate at which susceptible individuals become infected, began to decline roughly 6 years after disease appearance, and that devils that get infected with DFTD are otherwise more fit than those that do not. Additionally, survival after infection is 12-24 months, longer than previous field estimates of 3-9 months based on recapture data (Wells et al. 2017). Two epidemiological models at different spatial scales (Siska et al. 2018; Wells et al. 2019) also predict persistence of devils over a wide range of scenarios, without explicitly incorporating genetic changes. Wells et al. (2019) predict devil persistence with or without DFTD in single populations, by including tumor growth and individual variation in tumor load, in approximately $80 \%$ of simulations. At the metapopulation level, Siska et al. (2018) predict dynamic, long-term coexistence of devils and DFTD, using models that incorporate spatial movement, local extinction, and recolonization.

\section{Current conservation strategies for Tasmanian devils}

There are several strategies that have been proposed to manage the impact of DFTD on wild Tasmanian devil populations (Jones et al. 2007; McCallum 2008), although some have proven ineffective or unfeasible. Removing diseased animals, or culling, was unsuccessful when attempted (Lachish et al. 2010), and models have shown that no feasible culling strategy exists because of the need for unachievably high capture rates (Beeton and McCallum 2011). Isolation of a disease-free population, for instance on islands or by fencing, has also been proposed (McCallum and Jones 2010; Huxtable et al. 2015). However, fencing and related strategies are impractical because devils form a relatively continuous metapopulation across the species range (Hendricks et al. 2017) with few natural barriers (Storfer et al. 2017), and DFTD has spread across nearly the entire topographically rugged range with few remaining diseasefree populations. As a result, two primary conservation strategies are currently being pursued: captive breeding and release, and vaccine development.

First, a captive insurance metapopulation distributed across a number of locations was established in 2006 with the goal of maintaining a disease-free population that is "genetically representative of the species" (CBSG/DPIPWE/ ARAZPA 2009). The insurance population has been managed using a pedigree approach geared to maximize genetic diversity across the genome (Hogg et al. 2015; Grueber et al. 2018). Genetic marker panels have been developed to monitor genetic diversity (Wright et al. 2015), and genetic data may provide more accurate information than pedigree estimates to minimize inbreeding and loss of variation (Hogg et al. 2018). A disease-free population was established on Maria Island, derived from the captive insurance population (Thalmann et al. 2016). McLennan et al. (2018) estimated a pedigree for this population based on a panel of microsatellite loci and predicted substantial loss of genetic variation, suggesting that the small size of this population limits its utility for long-term conservation efforts in the absence of continued supplementation. A disease-free population was also established on the Forestier Peninsula, which is connected to mainland Tasmania by a road bridge across a human-made canal. Devils were first eradicated from the 64,000 hectare peninsula and then disease-free individuals introduced from the captive population (Huxtable et al. 2015). Despite the use of genetic markers to quantify variation and estimate pedigree relationships, genetic management of the captive population has not focused specifically on loci associated with DFTD.

An original goal of the insurance population was to be a source for reintroduction following local extinction of devil populations (CBSG/DPIPWE/ARAZPA 2009), although no local extinctions have since been documented. Nonetheless the Tasmanian government has released more than 80 animals from the insurance population into the wild, with the stated objectives of boosting devil population size and increasing genetic diversity (DPIPWE 2018). Behavioral changes and possibly domestication selection have reduced the fitness of individuals released from the insurance population, including increased vulnerability to vehicle mortality (Grueber et al. 2017). Currently, genetic variation at specific loci showing signatures of selection in response to DFTD, or associated with DFTD-related phenotypes such as tumor regression, is not considered in assessing individuals for release into wild populations.

Second, researchers are investigating the development of a vaccine that could be delivered to wild devils. The prospect of success is supported by progress in understanding immune responses in devils (Brown et al. 2011, 2016; Kreiss et al. 2015) and the observations of tumor regression 
and immune response in wild populations described above (Pye et al. 2016a). Experimentally treating devils with radiation-killed DFTD cells can produce a detectable immune response, which in some cases led to tumor regression in captive animals (Tovar et al. 2017). Pye et al. (2018) found that devils treated with DFTD cells manipulated to produce MHC class I antigens induce an antibody response in devils released into the wild. Yet despite this progress, it is not yet known whether immunization may be protective against DFTD infection in the field.

\section{Genetic management and future directions}

Current conservation efforts in Tasmanian devils are geared toward maintenance of overall genetic diversity in captivity, to release of individuals into existing wild populations to supplement overall genetic variation and demographic population sizes, and to mitigate the extent of ecosystem change due to trophic cascades (CBSG/DPIPWE/ARAZPA 2009; Huxtable et al. 2015). However, devils have the potential to be a model system for designing conservation strategies focused on adaptive genetic diversity. Devils have been the subject of long-term mark-recapture studies throughout the species range, with phenotypic data and tissue samples collected from over 10,000 individuals in addition to over 2000 tumor biopsies. Traditional genetic tools such as Sanger sequencing (Cheng et al. 2012) and microsatellite loci (Jones et al. 2003) have been widely applied, and microsatellites are used in genetic monitoring and pedigree estimation in the captive population (Hogg et al. 2015, 2017). Genomic tools include devil reference genome and transcriptome sequences (Murchison et al. 2010), tumor genome sequences (Stammnitz et al. 2018), comparative genomics (Wright et al. 2017; Margres et al. 2018b), and a number of genetic marker panels (Siddle et al. 2010; Morris et al. 2015; Wright et al. 2015; Epstein et al. 2016; Margres et al. 2018a). Given these resources and substantial novel results, it is timely to re-evaluate a few basic questions about strategies for devil conservation: What is likely to happen to devil populations in the absence of management actions? What are the benefits and risks of the ongoing management actions on devil populations, and how do they change predicted future outcomes? What is the best management strategy to maintain adaptive potential in devils, in the face of DFTD as well as other environmental changes, into the future?

Recent results point toward greater likelihood of longterm devil persistence than previously expected without any intervention. This conclusion comes from predictive modeling (Wells et al. 2017, 2019; Siska et al. 2018) as well as evidence of DFTD-relevant genetic variation (Margres et al. 2018a), recovery of DFTD-infected individuals (Pye et al. 2016a; Wright et al. 2017; Margres et al. 2018b), shifts in life history traits (Jones et al. 2008; Hamede et al. 2012; Lazenby et al. 2018; Russell et al. 2018), and rapid evolution at the genomic level in response to DFTD (Epstein et al. 2016; Hubert et al. 2018). This provides some margin of time to optimize conservation strategies with regard to adaptive genetic variation. Note, however, that the same models predict long-term persistence of devils at much lower population densities, particularly under scenarios in which DFTD also persists (Siska et al. 2018; Wells et al. 2019). Thus in the absence of any conservation actions, devils are likely to persist, but active management may be necessary if a conservation goal is to restore population densities and the ecological function of devils as the current apex mammalian predator and key scavenger (Hollings et al. 2015; Cunningham et al. 2018). Future research priorities include incorporating genetic variation and evolution in both devils and DFTD into predictive models, as well as empirically investigating coevolution between host and tumor.

Release of individuals from captivity into wild populations has the potential benefits of increasing numbers of individuals in these populations (i.e. demographic rescue), as well as increasing adaptive genetic diversity, potentially improving population fitness through genetic rescue. Attempts at genetic rescue carry the risk of outbreeding depression-reduced fitness as a result of erosion of locally adapted genotypes. Recent work shows that genetic rescue attempts often do not suffer from outbreeding depression and increase fitness (Frankham 2015; Whiteley et al. 2015). A notable exception to this trend is when captive populations are used as a source for translocation, because of the potentially rapid and unavoidable genetic changes that can occur in captivity (Whiteley et al. 2015). Additionally, genetic rescue is only appropriate if there is evidence of inbreeding depression or reduced fitness due to low genetic diversity (Weeks et al. 2011; Whiteley et al. 2015). While devils have low genetic diversity and some populations have shown declines (Farquharson et al. 2018), there is no evidence for inbreeding depression in wild (Brüniche-Olsen et al. 2013) or captive (Gooley et al. 2017) populations, or genetic limits on devil population fitness.

Recent genomic and modeling results in devils discussed above suggest that the risks to release of captive individuals into natural populations may be substantial, and we argue against further release of captive individuals into DFTD-infected wild populations. Translocated individuals can carry deleterious alleles and counteract the effects of natural selection (for example in a salmonid fish; Ferchaud et al. 2018). In the case of devil populations this includes increasing the frequency of alleles conferring susceptibility to DFTD. Moreover, increasing the proportion or number of susceptible individuals would change the dynamics of the DFTD epidemic, increasing the force of infection and disease transmission rates, potentially outstripping any 
demographic or genetic gains (Wells et al. 2017). Because the insurance population was drawn from DFTD-free populations, with limited supplementation with orphans from diseased populations, and has not been managed for genetic factors explicitly related to DFTD, it is reasonable to assume that the captive population has higher frequencies of susceptible genotypes than natural populations that have evolved in response to DFTD (Epstein et al. 2016). As a result, perhaps counterintuitively, increasing natural population sizes in the short term with supplementation of captive individuals could have the long-term consequence of reducing population sizes because of changes in disease dynamics.

We recommend genetic monitoring of allelic variation at DFTD-related loci in both captive and wild populations, as these loci continue to be identified. This would inform predictions about the future of the epidemic, as well as determining how the frequency of susceptible individuals would be affected by supplementation. Development of a panel of genetic markers that would predict individual-level susceptibility would also provide a tool for guiding captive breeding efforts toward natural devil populations that have the capacity to adapt to DFTD, and choosing individual devils for possible translocation from the captive population to the wild. To date, most loci identified as candidates from the selection and association tests described above (Table S1) have not been functionally validated, for instance by study of gene expression and function with transcriptomics, genetic manipulation in vitro, or other approaches. We do not advocate selecting for particular genotypes at a set of loci based on these published candidates. But as our understanding improves, management could include the goal of maintaining genetic variation at these loci where strong evidence for an association with DFTD-related phenotypes is found, even if the complete mechanistic link remains unclear. Over the longer term, better understanding of the relationships among genetic variation, individual susceptibility, and populationlevel disease outcomes will inform other genetic management strategies. One important result of the predictive modeling and evidence for rapid evolution described above is that it may provide somewhat less urgency for active management, as unmanaged natural populations may be more likely to persist, although at lower densities, than previously predicted. We suggest that this time window should be used to evaluate potential genetic management strategies targeting DFTD-associated loci, as our understanding of these loci continues to improve.

However, a risk of managing for DFTD-related genetic variation is adverse effects on variation important for other population stressors, such as habitat loss, anthropogenic mortality, or environmental change, or other pathogens. Remarkably, a second independent transmissible cancer was recently discovered in Tasmanian devils, called DFT2 (Pye et al. 2016b), suggesting that devils may be particularly susceptible to this unique type of disease. DFT2 appears to have broad similarities to DFTD (Stammnitz et al. 2018), suggesting that similar suites of genes involved in immune system function and cancer may be involved in potential resistance and evolutionary response to both diseases. The appearance of DFT2 raises the possibility of discovering and managing devil populations for genetic factors associated with resistance or other phenotypes associated with transmissible cancers in general, rather than DFTD specifically, but to date any host responses to DFT2 remain poorly understood.

Tasmanian devils face a unique conservation threat in the form of transmissible cancer, but the vast genetic resources available for the devil-DFTD system make the devil a potential model system for management of adaptive genetic variation. We urge continual re-evaluation of devil conservation strategies as our understanding advances. More broadly, the devil-DFTD system illustrates how modern advances in genomics can allow detection of adaptive or functionally significant loci in species of conservation concern, including loci associated with an acute threat to species persistence, such as disease. Identification of such loci should lead conservation biologists to consider the effects of current conservation strategies on this adaptive variation, and the potential utility of genetic management strategies for endangered populations beyond simple maximization of genetic diversity.

Acknowledgements This work was supported by NSF Grant DEB1316549 and NIH Grant R01-GM126563 as part of the joint NSFNIH-USDA Ecology and Evolution of Infectious Diseases program.

\section{References}

Bay RA, Harrigan RJ, Underwood VL, Gibbs HL, Smith TB, Ruegg K (2018) Genomic signals of selection predict climate-driven population declines in a migratory bird. Science 359:83-86

Beeton N, McCallum H (2011) Models predict that culling is not a feasible strategy to prevent extinction of Tasmanian devils from facial tumour disease. J Appl Ecol 48:1315-1323

Brown GK, Kreiss A, Lyons AB, Woods GM (2011) Natural killer cell mediated cytotoxic responses in the Tasmanian Devil. PLoS ONE 6:e24475

Brown GK, Tovar C, Cooray AA, Kreiss A, Darby J, Murphy JM, Corcoran LM, Bettiol SS, Lyons AB, Woods GM (2016) Mitogen-activated Tasmanian devil blood mononuclear cells kill devil facial tumour disease cells. Immun Cell Biol 94:673-679

Brüniche-Olsen A, Burridge CP, Austin JJ, Jones ME (2013) Disease induced changes in gene flow patterns among Tasmanian devil populations. Biol Conserv 165:69-78

Brüniche-Olsen A, Jones ME, Austin JJ, Burridge CP, Holland BR (2014) Extensive population decline in the Tasmanian devil predates European settlement and devil facial tumour disease. Biol Lett 10:20140619

Brüniche-Olsen A, Jones ME, Burridge CP, Murchison EP, Holland BR, Austin JJ (2018) Ancient DNA tracks the mainland extinction and island survival of the Tasmanian devil. J Biogeogr 2014:1-14 
CBSG/DPIPWE/ARAZPA (2009) The save the Tasmanian Devil Program: strategic framework for an insurance meta-population. Department of Primary Industries, Parks, Water and Environment, Hobart

Cheng Y, Sanderson C, Jones M, Belov K (2012) Low MHC class II diversity in the Tasmanian devil (Sarcophilus harrisii). Immunogenetics 64:525-533

Cunningham CX, Johnson CN, Barmuta LA, Hollings T, Woehler EJ, Jones ME (2018) Top carnivore decline has cascading effects on scavengers and carrion persistence. Proc R Soc B 285:20181582

DPIPWE (2018) Wild devil recovery project. Tasmanian Government. Department of Primary Industries, Parks, Water and Environment, Tasmania. https://dpipwe.tas.gov.au/wildlife-manag ement/save-the-tasmanian-devil-program/about-the-program/ wild-devil-recovery-project. Accessed 6 Sept 2018

Epstein B, Jones ME, Hamede R et al (2016) Rapid evolutionary response to a transmissible cancer in Tasmanian devils. Nat Commun 7:12684

Farquharson KA, Gooley RM, Fox S et al (2018) Are any populations "safe"? Unexpected reproductive decline in a population of Tasmanian devils free of devil facial tumour disease. Wildl Res 45:31-37

Ferchaud AL, Laporte M, Perrier C, Bernatchez L (2018) Impact of supplementation on deleterious mutation distribution in an exploited salmonid. Evol Appl 11:1053-1065

Frankham R (2015) Genetic rescue of small inbred populations: meta-analysis reveals large and consistent benefits of gene flow. Mol Ecol 24:2610-2618

Gilpin ME, Soulé ME (1986) Minimum viable populations: processes of species extinction. In: Soule ME (ed) Conservation biology: the science of scarcity and diversity. Sinauer Associates, Sunderland, pp 19-34

Gooley R, Hogg CJ, Belov K, Grueber CE (2017) No evidence of inbreeding depression in a Tasmanian devil insurance population despite significant variation in inbreeding. Sci Rep 7:1830

Grueber CE, Reid-Wainscoat EE, Fox S et al (2017) Increasing generations in captivity is associated with increased vulnerability of Tasmanian devils to vehicle strike following release to the wild. Sci Rep 7:2161

Grueber CE, Peel E, Wright B, Hogg CJ, Belov K (2018) A Tasmanian devil breeding program to support wild recovery. Reprod Fertil Dev. https://doi.org/10.1071/RD18152

Hamede R, Lachish S, Belov K (2012) Reduced effect of Tasmanian devil facial tumor disease at the disease front. Conserv Biol 26:124-134

Hamede RK, McCallum H, Jones M (2013) Biting injuries and transmission of Tasmanian devil facial tumour disease. J Anim Ecol $82: 182-190$

Hamede RK, Pearse A-M, Swift K, Barmuta LA, Murchison EP, Jones ME (2015) Transmissible cancer in Tasmanian devils: localized lineage replacement and host population response. Proc R Soc Lond B 282:20151468

Hendricks S, Epstein B, Schönfeld B et al (2017) Conservation implications of limited genetic diversity and population structure in Tasmanian devils (Sarcophilus harrisii). Conserv Genet 18:977-982

Hoban S, Kelley JL, Lotterhos KE et al (2016) Finding the genomic basis of local adaptation: pitfalls, practical solutions, and future directions. Am Nat 188:379-397

Hogg CJ, Ivy JA, Srb C et al (2015) Influence of genetic provenance and birth origin on productivity of the Tasmanian devil insurance population. Conserv Genet 16:1465-1473

Hogg CJ, Lee AV, Srb C, Hibbard C (2017) Metapopulation management of an endangered species with limited genetic diversity in the presence of disease: the Tasmanian devil Sarcophilus harrisii. Int Zoo Yearb 51:137-153
Hogg CJ, Wright B, Morris KM, Lee AV, Ivy JA, Grueber CE, Belov K (2018) Founder relationships and conservation management: empirical kinships reveal the effect on breeding programmes when founders are assumed to be unrelated. Anim Conserv. https://doi.org/10.1111/acv.12463

Hollings T, McCallum H, Kreger K, Mooney N, Jones M (2015) Relaxation of risk-sensitive behaviour of prey following disease-induced decline of an apex predator, the Tasmanian devil. Proc R Soc B 282:20150124

Hubert J-N, Zerjal T, Hospital F (2018) Cancer- and behavior-related genes are targeted by selection in the Tasmanian devil (Sarcophilus harrisii). PLoS ONE 13:e0201838

Huxtable SJ, Lee DV, Wise P, Save the Devil Program (2015) Metapopulation management of an extreme disease scenario. In: Armstrong DP, Hayward MW, Moro D, Seddon PJ (eds) Advances in reintroduction biology of Australian and New Zealand Fauna. CSIRO Publishing, Melbourne, pp 141-154

Johnson WE, Onorato DP, Roelke ME et al (2010) Genetic restoration of the Florida panther. Science 329:1641-1645

Jones ME, Paetkau D, Geffen E, Mortiz C (2003) Microsatellites for the Tasmanian devil (Sarcophilus laniarius). Mol Ecol Notes 3:277-279

Jones ME, Paetkau D, Geffen ELI, Moritz C (2004) Genetic diversity and population structure of Tasmanian devils, the largest marsupial carnivore. Mol Ecol 13:2197-2209

Jones ME, Jarman PJ, Lees CM (2007) Conservation management of Tasmanian devils in the context of an emerging, extinctionthreatening disease: devil facial tumor disease. EcoHealth 4:326-337

Jones ME, Cockburn A, Hamede R et al (2008) Life-history change in disease-ravaged Tasmanian devil populations. Proc Natl Acad Sci USA 105:10023-10027

Kardos M, Taylor HR, Ellegren H, Luikart G, Allendorf FW (2016) Genomics advances the study of inbreeding depression in the wild. Evol Appl 9:1205-1218

Kreiss A, Brown GK, Tovar C, Lyons AB, Woods GM (2015) Evidence for induction of humoral and cytotoxic immune responses against devil facial tumor disease cells in Tasmanian devils (Sarcophilus harrisii) immunized with killed cell preparations. Vaccine 33:3016-3025

Lachish S, McCallum HI, Mann D, Pukk CE, Jones ME (2010) Evaluation of selective culling of infected individuals to control Tasmanian devil facial tumor disease. Conserv Biol 24:841-851

Lachish S, Miller KJ, Storfer A, Goldizen AW, Jones ME (2011) Evidence that disease-induced population decline changes genetic structure and alters dispersal patterns in the Tasmanian devil. Heredity 106:172

Lande R (1995) Mutation and conservation. Conserv Biol 9:782-791

Lazenby BT, Tobler MW, Brown WE et al (2018) Density trends and demographic signals uncover the long-term impact of transmissible cancer in Tasmanian devils. J Appl Ecol 55:1368-1379

Luikart G, Kardos M, Hand BK, Rajora OP, Aitken SN, Hohenlohe PA (2018) Population genomics: advancing understanding of nature. In: Rajora OP (ed) Population genomics: concepts, approaches, and applications. Springer, Cham

Margres MJ, Jones M, Epstein B et al (2018a) Large-effect loci affect survival in Tasmanian devils (Sarcophilus harrisii) infected with a transmissible cancer. Mol Ecol 27:4189-4199

Margres MJ, Ruiz-Aravena M, Hamede RK et al (2018b) The genomic basis of tumor regression in Tasmanian devils (Sarcophilus harrisii). Genom Biol Evol 10:3012-3025

McCallum H (2008) Tasmanian devil facial tumour disease: lessons for conservation biology. Trends Ecol Evol 23:631-637

McCallum H, Jones M (2010) Sins of omission and sins of commission: St Thomas Aquinas and the devil. Aust Zool 35:307-314 
McCallum H, Jones M, Hawkins C et al (2009) Transmission dynamics of Tasmanian devil facial tumor disease may lead to diseaseinduced extinction. Ecology 90:3379-3392

McLennan EA, Gooley RM, Wise P, Belov K, Hogg CJ, Grueber CE (2018) Pedigree reconstruction using molecular data reveals an early warning sign of gene diversity loss in an island population of Tasmanian devils (Sarcophilus harrisii). Conserv Genet $19: 439-450$

Miller W, Hayes VM, Ratan A et al (2011) Genetic diversity and population structure of the endangered marsupial Sarcophilus harrisii (Tasmanian devil). Proc Natl Acad Sci USA 108:12348-12353

Morris K, Austin JJ, Belov K (2013) Low major histocompatibility complex diversity in the Tasmanian devil predates European settlement and may explain susceptibility to disease epidemics. Biol Lett 9:20120900

Morris KM, Wright B, Grueber CE, Hogg C, Belov K (2015) Lack of genetic diversity across diverse immune genes in an endangered mammal, the Tasmanian devil (Sarcophilus harrisii). Mol Ecol 24:3860-3872

Murchison EP (2009) Clonally transmissible cancers in dogs and Tasmanian devils. Oncogene 27:S19

Murchison EP, Tovar C, Hsu A et al (2010) The Tasmanian devil transcriptome reveals Schwann cell origins of a clonally transmissible cancer. Science 327:84-87

Pearse AM, Swift K (2006) Allograft theory: transmission of devil facial-tumour disease. Nature 439:549

Pearse AM, Swift K, Hodson P, Hua B, McCallum H, Pyecroft S, Taylor R, Eldridge MD, Belov K (2012) Evolution in a transmissible cancer: a study of the chromosomal changes in devil facial tumor (DFT) as it spreads through the wild Tasmanian devil population. Cancer Genet 205:101-112

Pye R, Hamede R, Siddle HV et al (2016a) Demonstration of immune responses against devil facial tumour disease in wild Tasmanian devils. Biol Lett 12:20160553

Pye RJ, Pemberton D, Tovar C et al (2016b) A second transmissible cancer in Tasmanian devils. Proc Natl Acad Sci USA 113:374-379

Pye R, Patchett A, McLennan E et al (2018) Immunization strategies Producing a humoral igg immune response against Devil Facial Tumor Disease in the Majority of Tasmanian Devils Destined for Wild release. Front Immunol 19:259

Rellstab C, Gugerli F, Eckert AJ, Hancock AM, Holderegger R (2015) A practical guide to environmental association analysis in landscape genomics. Mol Ecol 24:4348-4370

Russell T, Madsen T, Thomas F, Raven N, Hamede R, Ujvari B (2018) Oncogenesis as a selective force: adaptive evolution in the face of a transmissible cancer. BioEssays 40:1700146

Santure AW, Garant D (2018) Wild GWAS-association mapping in natural populations. Mol Evol Res 18:729-738

Siddle HV, Marzec J, Cheng Y, Jones M, Belov K (2010) MHC gene copy number variation in Tasmanian devils: implications for the spread of a contagious cancer. Proc R Soc Lond B 277:2001-2006

Siddle HV, Kreiss A, Tovar C, Yuen CK, Cheng Y, Belov K, Swift K, Pearse AM, Hamede R, Jones ME, Skjødt K (2013) Reversible epigenetic down-regulation of MHC molecules by devil facial tumour disease illustrates immune escape by a contagious cancer. Proc Natl Acad Sci USA 110:5103-5108

Siska V, Eriksson A, Mehlig B, Manica A (2018) A metapopulation model of the spread of the Devil Facial Tumour Disease predicts the long term collapse of its host but not its extinction. arXiv: 1806.05449

Soulé ME, Wilcox B (1980) Conservation biology: an evolutionaryecological perspective. Oxford University Press, Oxford

Stammnitz MR, Coorens TH, Gori KC (2018) The origins and vulnerabilities of two transmissible cancers in Tasmanian devils. Cancer Cell 33:607-619

Storfer A, Epstein B, Jones M, Micheletti S, Spear SF, Lachish S, Fox $S$ (2017) Landscape genetics of the Tasmanian devil: implications for spread of an infectious cancer. Conserv Genet 18:1287-1297

Storfer A, Hohenlohe PA, Margres MJ et al (2018a) The devil is in the details: Genomics of transmissible cancers in Tasmanian devils. PLoS Pathog 14:e1007098

Storfer A, Patton A, Fraik AK (2018b) Navigating the interface between landscape genetics and landscape genomics. Front Genet 9:68

Thalmann S, Peck S, Wise P, Potts JM, Clarke J, Richley J (2016) Translocation of a top-order carnivore: tracking the initial survival, spatial movement, home-range establishment and habitat use of Tasmanian devils on Maria Island. Aust Mammal 38:68-79

Tovar C, Pye RJ, Kreiss A (2017) Regression of devil facial tumour disease following immunotherapy in immunised Tasmanian devils. Sci Rep 7:43827

Ujvari B, Pearse AM, Swift K, Hodson P, Hua B, Pyecroft S, Taylor R, Hamede R, Jones M, Belov K, Madsen T (2014) Anthropogenic selection enhances cancer evolution in Tasmanian devil tumours. Evol Appl 7:260-265

Weeks AR, Sgro CM, Young AG, Frankham R, Mitchell NJ, Miller KA, Byrne M, Coates DJ, Eldridge MD, Sunnucks P, Breed MF (2011) Assessing the benefits and risks of translocations in changing environments: a genetic perspective. Evol Appl 4:709-725

Wells K, Hamede R, Kerlin DH, Storfer A, Hohenlohe PA, Jones ME, McCallum HI (2017) Infection of the fittest: devil facial tumour disease has greatest effect on individuals with highest reproductive output. Ecol Lett 20:770-778

Wells K, Hamede RK, Jones ME, Hohenlohe PA, Storfer A, McCallum HI (2019) Individual and temporal variation in pathogen load predicts long-term impacts of an emerging infectious disease. Ecology. https://doi.org/10.1002/ecy.2613

Whiteley AR, Fitzpatrick SW, Funk WC, Tallmon DA (2015) Genetic rescue to the rescue. Trends Ecol Evol 30:42-49

Wright B, Morris K, Grueber CE, Willet CE, Gooley R, Hogg CJ, O’Meally D, Hamede R, Jones M, Wade C, Belov K (2015) Development of a SNP-based assay for measuring genetic diversity in the Tasmanian devil insurance population. BMC Genom $16: 791$

Wright B, Willet CE, Hamede R, Jones M, Belov K, Wade CM (2017) Variants in the host genome may inhibit tumour growth in devil facial tumours: evidence from genome-wide association. Sci Rep $7: 423$

Publisher's Note Springer Nature remains neutral with regard to jurisdictional claims in published maps and institutional affiliations. 